\title{
Proposing a New Algorithm for Solving Job Assignment Problem by Partition to Sub Matrices
}

\author{
Ruqayya Z. Sha'ban \\ Assistant lecturer \\ College of Medicine \\ University of Mosul
}

\begin{abstract}
A new algorithm has been developed by throughout this work in order to solve job assignment problems numerically. The algorithm is however suitable for small and big problems. The algorithm proves efficiency, because it eliminates big parts of possible outcome. The elimination of possible outcome increases with the size of problem. The Algorithm based on dividing the matrix into smaller matrices, then divided matrix into sub matrices. The formation of these small matrices in the bigger matrix is understood. Their places in the big matrix are also known.

\section{اقتراح خوارزمية جديدة لحل مسألة التخصيص بالتقسيم إلى مصفوفات فرعية \\ المستخلص}

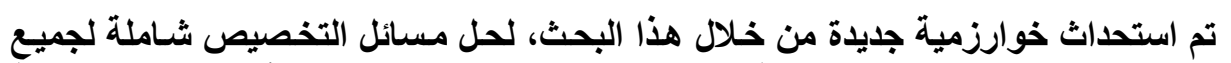

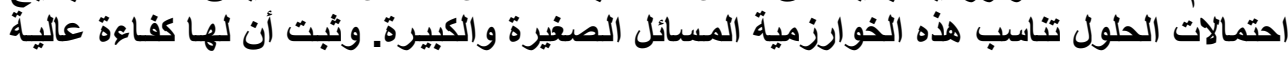

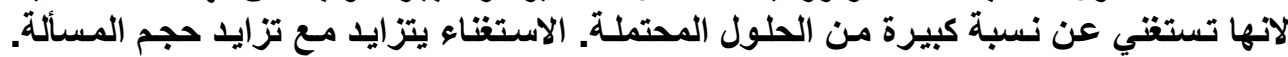

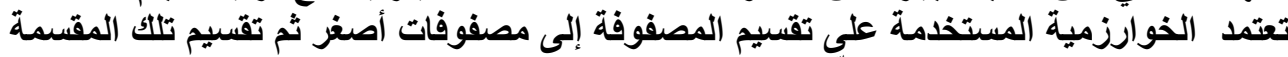

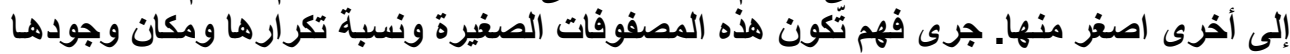

في المصفوفات الأكبر منها.
\end{abstract}

\section{Introduction}

Job assignment is a technique that can be used to solve problems of allocating resources, such as allocating jobs to machines (or to men). The allocation process, according to this technique, must provide sufficient number of jobs so each machine (or man) must have a single job; no more and no less. In addition to the objective function is given a minimum total assignment cost (عادل، وآخرون).

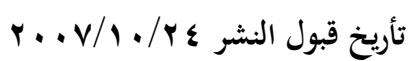

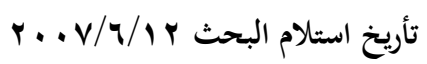


As for solving the current problem, a square matrix is placed. The matrix has a sort of rows that are equal to the number of machines and columns equal to the number of jobs. Each cell in this matrix shows the cost, which may occur if a machine is performing one of these jobs, as shown in Table (1), (Ackoff \& Sasieni, 1968; Taha, 2003;1999، علي وأخرون، Bilkent, via Internat)

Several methods are available for solving this type of problems. These methods are mostly manual proposed during the ages which proceeded the age of computers. Recently, methods have been adapted to computers, so they can be solved more easily and quickly.

\begin{tabular}{|c|c|c|c|c|c|c|c|}
\hline $\mathrm{Job}_{\mathrm{i}}$ & $\mathrm{Job}_{1}$ & $\mathrm{Job}_{2}$ & Job & & \multicolumn{2}{|c|}{$\mathrm{Job}_{\mathrm{n}}$} & \\
\hline Machine $_{j}$ & 1 & 2 & - & - & - & $\mathrm{n}$ & \\
\hline Machine $_{1}$ & $\mathrm{C}_{11}$ & $\mathrm{C}_{12}$ & & & & $\mathrm{C}_{1 \mathrm{n}}$ & $\mathrm{a}_{1}=1$ \\
\hline- & $\mathrm{C}_{21}$ & $\mathrm{C}_{22}$ & & & & $\mathrm{C}_{2 \mathrm{n}}$ & $a_{2}=1$ \\
\hline- & - & - & & & & - & - \\
\hline Machine $_{m}$ & $\mathrm{C}_{\mathrm{m} 1}$ & $\mathrm{C}_{\mathrm{m} 2}$ & & & & $\mathrm{C}_{\mathrm{mn}}$ & $a_{m}=1$ \\
\hline$b_{j}$ & $b_{1}=1$ & $\mathrm{~b}_{2}=1$ & & & & $b_{n}=1$ & \\
\hline
\end{tabular}

Table 1

\section{The Assignment}

In this table (n) must be equal (m); the Mathematical Model of the problem as following (النعيمي، (1999).

(1) $X_{i j}=\left\{\begin{array}{l}1 \text { if Job (i) is assign to machine ( } j) \\ 0 \text { otherwise }\end{array}\right.$

(2) Min,Max Z= $\sum_{i=1}^{n} \sum_{j=1}^{m} C_{i j} X_{i j}$ objective function

Subject to:

(3) $\sum_{i=1}^{n} \mathrm{X}_{\mathrm{ij}}=1$ for $j=1,2, \ldots \ldots, n$

(4)

$$
\begin{gathered}
\sum_{j=1}^{m} \mathrm{X}_{i j}=1 \text { for } i=1,2, \ldots \ldots, m \\
\mathrm{X}_{\mathrm{ij}}=0 \text { or } 1 \text { for all } i \text { and } j
\end{gathered}
$$


The adaptation operation may not lead to the desired target. For example, one of these methods is based, as part of the solution, on covering the zeros in the matrix by minimum number of lines.

This coverage can be done by a computer program, where the first line covers as much as the zeros and the second line covers as much as possible of the uncovered zeros and so for the rest of the zeros. In this method, a big problem can be solved by computer in a quite short time. Unfortunately, starting with a line which covers the largest number of zeros may not lead to the minimum number of lines. Consequently, the optimal solution, for some cases, could fail (Winston, 1994).

On the other hand, enumerated method is a method which is based on calculating all possible solutions. Among them the minimum cost would be found. As for the problem of job assignment, the cost matrix of reasonable size could have million of different outcomes. They are estimated by the factorial number of the rows (or columns) of the matrix. For instance, the matrix of $3 \times 3$ has six different solutions. While, the matrix of $5 \times 5$ has 120 and the matrix of $10 \times 10$ have more than three and half million (3628800)

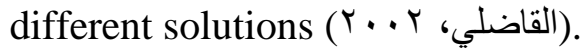

Despite this big job on computer, someone may think that this approach looks perfect and an easy task for computer, especially on fast computers. It has found that this method is time consuming, impractical and difficult to be achieved. Therefore, it would better to propose new methods which can help in eliminating part of the outcomes. Hence, the solution could be obtained in reasonable computer effort.

The objective of this work is to develop an algorithm which can replace the enumerating method and reduce the effort that computer should make for solving this problem. The characteristics of the assignment matrix have been studied and accordingly the algorithm is proposed.

\section{The Properties of the Matrix for Job Assignment}

The known methods which have been found in literature take the assignment matrix as a single matrix which can not be divided into sub matrix. In the current work, the matrix is studied as one which can be divided into several sub matrices and sub sub submatrices. The objective behind this division is to solve these small matrices as steps to solve the problem matrix .This approach facilitate the understanding process of the nature of the matrix and to propose an algorithm which can determine the solution of this problem accurately and efficiently. The investigations taken place in this work, concerning the square matrix of job assignment have proved the following:

1. The matrix of (n) dimension has (n) different sub matrix of (n-1) dimension. Consequently, each matrix of (n-1) dimension has (n-1) different sub submatrix of (n-2) dimension. This is true for all other 
smaller matrices till the smallest matrix of two dimensions, as the matrix of 3 dimensions has three different $(2 \times 2)$ sub matrixes.

2. Every small matrix is found in several bigger sub matrix.

3. Each sub matrix of fixed dimension is found equally distributed in the bigger sub matrices. No sub matrix has found in different rate than the others of the same dimension.

4. The matrix has all the possible smaller sub matrix starting from (n-1xn-1) to $(2 \times 2)$ sub matrices.

5. From the definition of combinations rule if there was (n) of elements and we need to take (s) element in each time where (s) represent any choosing to the number of (s) from elements with don't care about the order method, for that the number of the sub matrix can be determined according to this equations:

$$
C_{s}^{n}=\frac{n !}{s ! *(n-s) !} \quad \text { where } s=\text { The size of sub matrix }
$$

This means if we have (nxn) size of problem then to create problem as size

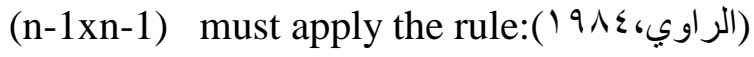

$m=C_{n-1}^{n}=\frac{n !}{(n-1) ! *(n-(n-1)) !} \quad \begin{aligned} & \text { Where } \mathrm{m}=\text { then number of submatrix } \\ & \text { have size }(\mathrm{n}-1 \mathrm{xn}-1)\end{aligned}$

\section{The Proposed Algorithm}

Step1:

Initialize the matrix of the main problem size ( $\mathrm{nxn})$. Let $\mathrm{x}=2$ be an initial value to number of the problems size of matrix) that must be annexed for each matrix.

Step2:

Create number of problems has size $(2 \times 2)$ matrix by select from the last two rows (i.e. row $n$ and row $n-1$ ) all the possible matrix of $2 x$ 2 as in Fig.(1). The number of these small matrices is estimated as relative to the number of rows (or columns) by this equation:

$\mathbf{n} ! /((\mathbf{n}-2) ! * 2 !)$. According to this equation, the $4 \times 4$ matrix has six $2 \times 2$ matrices and $10 \times 10$ matrix has forty five $2 \times 2$ matrices. 

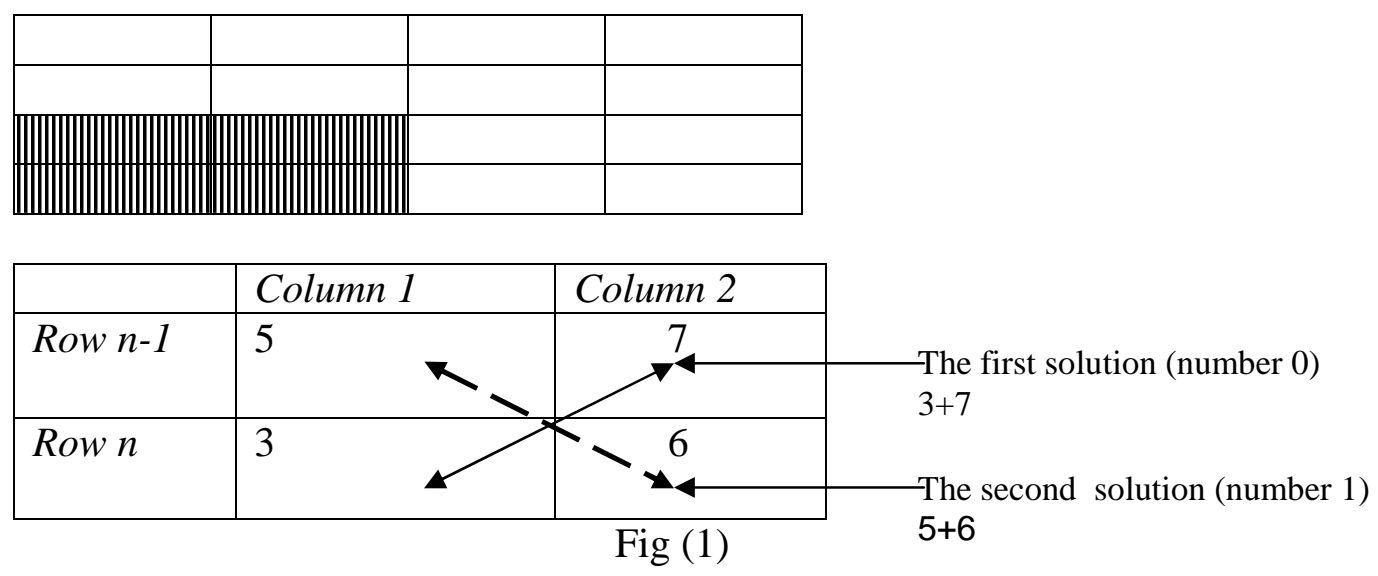

The $2 \times 2$ matrix has just two possible solutions. Its minimum solution is determined. For instance, the 2 × 2 matrix has two solutions:

The first solution (number 0) is 11 and the second solution (number 1) is 10 . A record is created to hold the detail of these calculations as in Fig.2. The record contains the column numbers from which this matrix is derived, the minimum solution and the number of the solution ( 0 or 1$)$.

\begin{tabular}{|c|c|c|c|}
\hline $\begin{array}{c}\text { Column number } \\
\text { of first column }\end{array}$ & $\begin{array}{c}\text { Column number } \\
\text { of second } \\
\text { column }\end{array}$ & $\begin{array}{c}\text { Number of } \\
\text { solution }\end{array}$ & Minimum solution \\
\hline
\end{tabular}

Fig 2

Record (1) of step 2

\section{Step 3:}

Each cell in the row ( $\mathrm{n}-\mathrm{x}$ ) (except two) is annexed to all the $2 \times 2$ matrices (see Fig. 3) which are formed in steps 2. The excluded two cells are belonging to the columns from which the $(2 \times 2)$ matrix is taken. The annexation process produces $(\mathrm{n}-\mathrm{x})$ sub matrix. For each matrix $(\mathrm{X} \times \mathrm{X})$ matrix, which is produced by this step, a record is created Fig. 3. The created records contain the following details:

1. The columns number of the $2 \times 2$ matrix

2. The column number from which the annexed cell is taken

3 . The solution number of $2 \times 2$ matrix; and

4. The cost at this stage which is the sum of the solution of the $2 \times 2$ matrix and the cost which is hold in the annexed cells. 


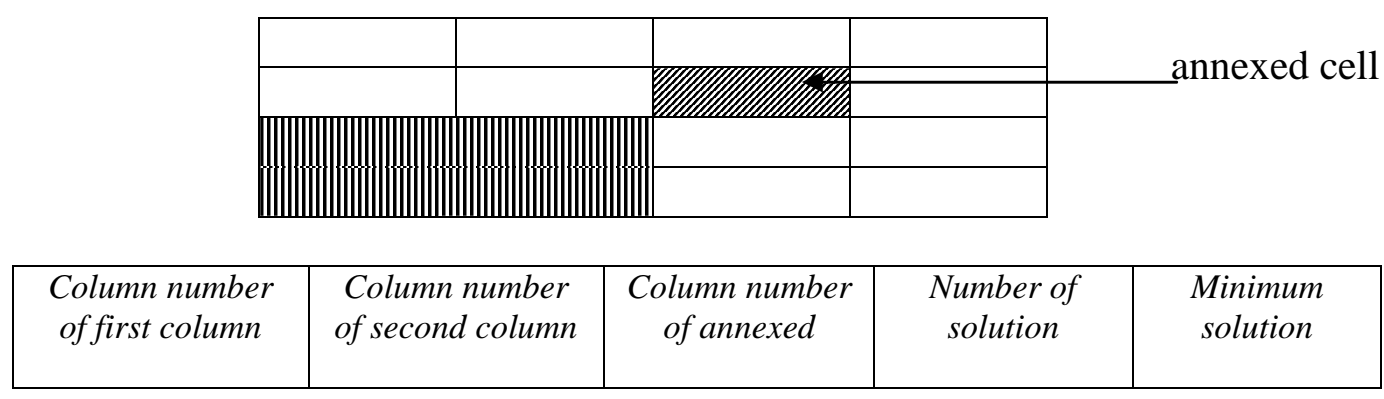

Fig.3

The form of matrix and Record (2) of step 3

Step 4:

The records from step 3 are including three columns number: the columns from which the matrix are taken and the column from which the annexed cell are chosen. Three records will be found carrying the same column number. These three records are compound together to forms the $3 \mathrm{x}$ 3 sub matrix of the problems. Among the three costs which are brought with these three records the minimum cost is selected to form the solution of the sub matrix, this is the best cost. A record for each $3 \times 3$, i.e. $(\mathrm{X} \times \mathrm{X})$ matrix is created to hold the details which identify this matrix.

Each record will includes

1. The three column number

2. The minimum cost (the best cost).

3. The identity which helps in identifying which one of the three records has the minimum cost.

Step 5: Increase value of $\mathrm{x}$ by $\mathrm{x}=\mathrm{x}+1$

\section{Step 6:}

Each $3 \times 3$ matrix must annexed to the cells from the row above that matrix. Exactly as it has done with $2 \times 2$ matrix. The same operations are done as steps $(3,4,5)$. These operations will result $4 \times 4$ matrices. These operations is repeated to form $5 \times 5$ matrices and so on until arrive up to the first column. These operations will give the optimum solution of the problem together with the path which gives this solution.

Step7: Draw the path which gives the optimal solution.

Step8: End 


\section{Attentions on Algorithm}

In this paper, a new algorithm has been proposed to solve the job assignment problems numerically. The solutions obtained by the new algorithm are eliminated by more of possible outcome; this is clear in the steps of algorithms as in the following:

\section{In Step 2:}

In this step, the number of possible solutions of the general problems reduced by $\% 50$. This is because the selections which are made at this step to eliminate one of the two possible outcomes of this small matrix.

\section{In Step 3:}

This step will reduce the number of possible outcomes to one third of the reaming $\% 50$ of the possible outcome which remains in step 2., i.e. about $\% 33.3$ of the remaining possible outcome solutions are eliminated at the end of this step and \% 16.7 will still exist. This means that this stage \% 83.3 of the total possible outcome is eliminated by $\% 16.7$, requires more analysis. For the general problem \% 95.8 of the total possible outcome is eliminated and just \% 4.2 of the possible solutions are remaining. 

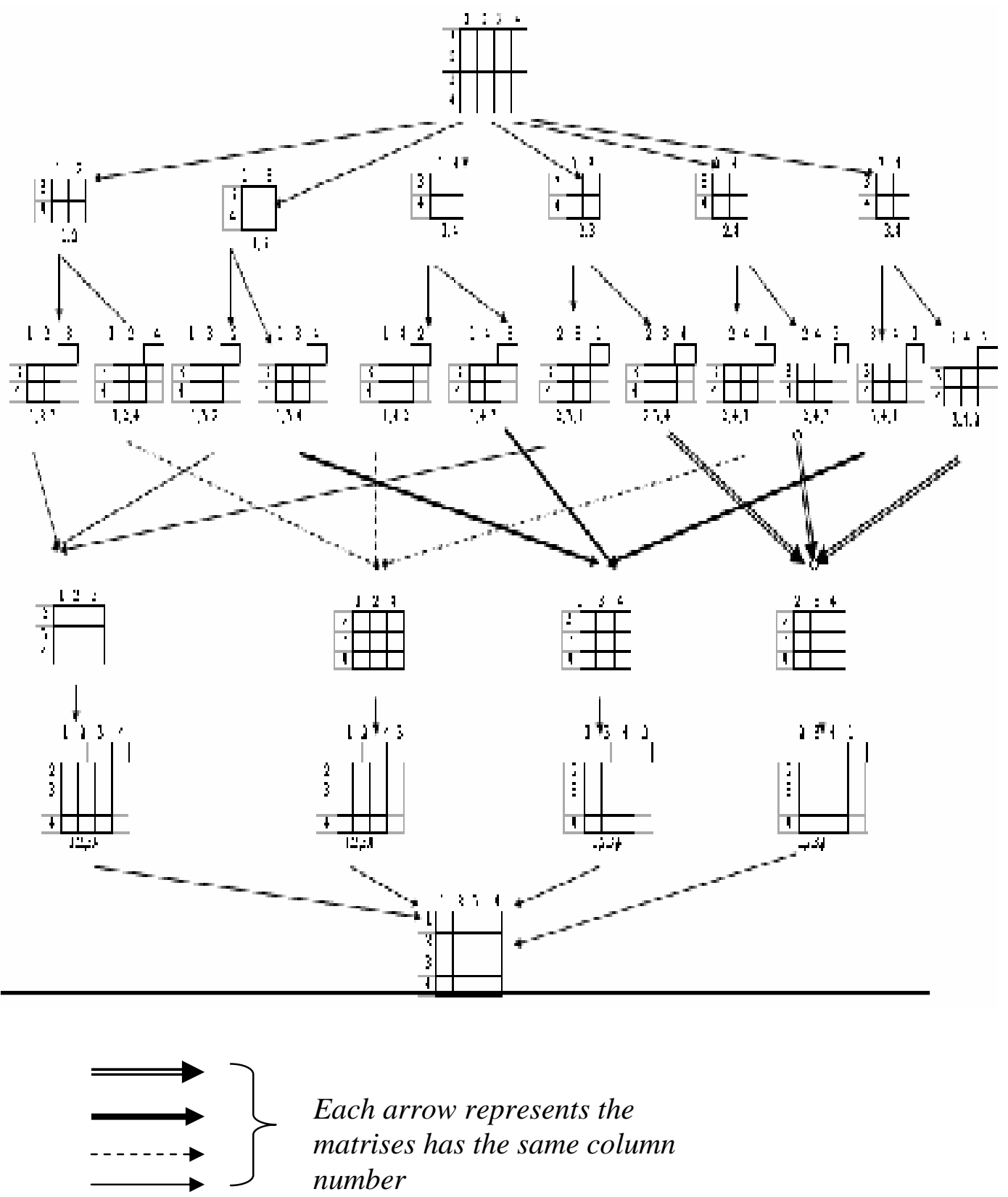

Each arrow represents the matrises has the same column number

Fig. 4

Solving Problem Consist Of Four Rows And Four Columns 


\section{Results and Calculations}

The following examples are written to illustrate the algorithms. Steps and tables about executing the algorithm and results of the example $4 \times 4$ are explained in (Appendix A).

The proposal algorithm wrote in software MATLAB version 6.5.0 in Pentium (4) processor 2.0GHz, see (Appendix B).

\section{First problem}

Figure (4) shows an example of $(4 \mathrm{x} 4)$ problem. This example shows that the problem has six $(2 \times 2)$ sub matrix. These sub matrices are derived from the rows four and three. Each sub matrix is solved according to step (1) and then annexed to a cell from the second row. The annexation process will be repeated twice for each $(2 \times 2)$ sub matrix. This will provide twelve forms as matrix of $(2 \times 2)$ tied to a cell from the second row. Each three of these forms will be combined together to form four $(3 \times 3)$ sub matrix. Each one of the four $(3 \times 3)$ sub matrix will be annexed to a cell from the first row.

These actions will give four of this form. These later four forms will be used to solve the $(4 \times 4)$ matrix. This later solution forms the final optimal solution. The path of the solution is obtained according to what is written in the records.

\section{Second problem}

\begin{tabular}{|c|c|}
\hline matrix & Number of sub matrix \\
\hline $2 \times 2$ & 10 \\
\hline $3 \times 3$ & 10 \\
\hline $4 \times 4$ & 5 \\
\hline $5 \times 5$ & 1 \\
\hline
\end{tabular}

The $(5 \times 5)$ problem has ten $(2 \times 2)$ sub matrices which are taken from the fifth and fourth rows. Each sub matrix is solved according to step (1) and then annexed to a cell from the third row. The annexation process will be repeated three times for each $(2 \times 2)$ sub matrix. This will provide thirty forms as matrix of $(2 \times 2)$ tied to a cell from the third row. Each three of these forms will have similar column numbers. They are combined together to form ten $(3 \times 3)$ sub matrix. Each $(3 \times 3)$ sub matrix will be annexed to two cells from the second row. This action will give twenty of this form. These later twenty forms will be used to form five $(4 \times 4)$ sub matrix. Each one of these later sub matrix will be annexed to a cell from the first row. These later five forms will give the final optimal solution $(5 \times 5)$ matrix. 


\section{Third Problem}

\begin{tabular}{|l|c|}
\hline Matrix & Number of sub matrix \\
\hline $2 \times 2$ & 45 \\
\hline $3 \times 3$ & 120 \\
\hline $4 \times 4$ & 210 \\
\hline $5 \times 5$ & 252 \\
\hline $6 \times 6$ & 210 \\
\hline $7 \times 7$ & 120 \\
\hline $8 \times 8$ & 45 \\
\hline $9 \times 9$ & 10 \\
\hline $10 \times 10$ & 1 \\
\hline
\end{tabular}

When a problem of $10 \times 10$ is solved by this method, forty five $(2 \times 2)$ sub matrices are going to form. Each one of them will be subjected to eight annexation process from cells in the eighth row. This later process will form three hundred sixty forms. They will be used to form hundred twenty $(3 \times 3)$ sub matrix.

The annexation process of $(3 \times 3)$ sub matrix with seven cells from the sixth row will produce eight hundred forty forms. Each four of them will be used to form $(4 \times 4)$ sub matrix. The number of formed $(4 \times 4)$ sub matrix is two hundred ten. The two hundred fifty two $(5 \times 5)$ sub matrix is formed from one thousand two hundred sixty annexed (4 x 4) sub matrix. Later all these $(5 \times 5)$ sub matrices will be formed 1260 annexes which consequently will form $210(6 \times 6)$ sub matrix. The procedure will continue on these later sub matrices to form $120(7 \times 7)$ sub matrix. The procedure would continue to form $45(8 \times 8)$ sub matrix. From them ten $(9 \times 9)$ sub matrix will formed, and from these later 10 matrix the optimal solution of the final $10 \times 10$ matrix will be known.

\section{أولاًَ- المراجع باللغة الاجنبية}

1. Ackoff, R. L. and Sasieni, M.W fundamentals of Operations Research, 1968, John Wiley and Sons, Inc.

2. Bilkent ,Operation Research Chapter 5: Transportation and Assingment Problems, Site from Internet, http://www.ie.bilkent.edu.tr/ ie202c/ch5.html-74k.

3. Taha, Hamdy A., Operations Research :An Introduction $7^{\text {th }}$ ed, 2003, By Pearson Education International, Inc.

4. Winston ,Wayne L.,Operations Research :Applications and Algorithms $3^{\text {rd }}$ ed ,1994, An imprint of Wadsworth Publishing Company Belmont, California. 


\section{ثانياً المراجع باللغة العربية}

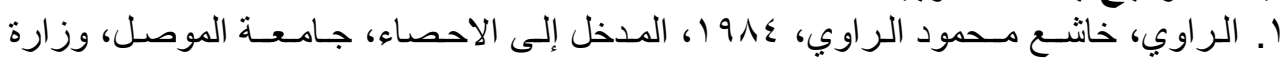

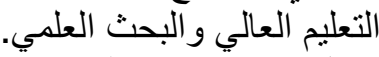

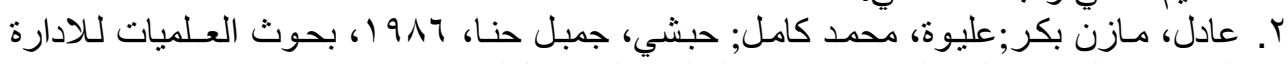

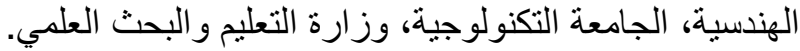

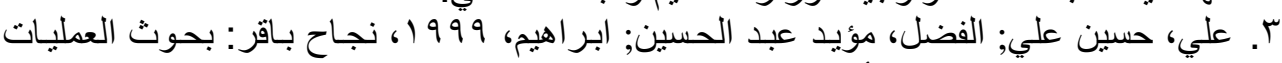

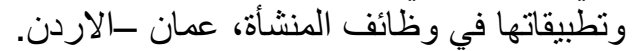

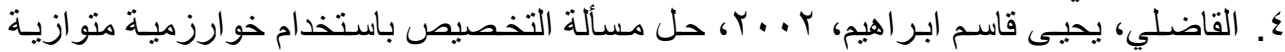

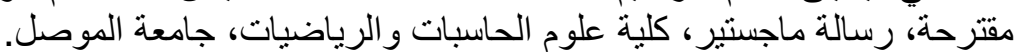

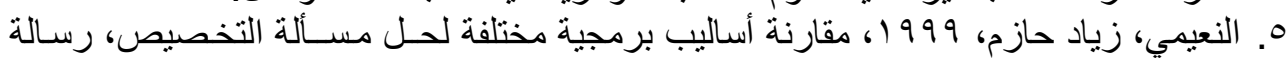
ماجستير، كلية علوم الحاسبات و الرياضيات، جارية جامعة الموصل بردئ. 